\title{
PCV2 trigger apoptosis of PK-15 cells through the PLC-IP3R-Ca2+ signaling pathway
}

\author{
Shuo Wang ${ }^{1}$, Chen $\mathrm{Li}^{2}$, Panpan $\mathrm{Sun}^{3}$, Jianli $\mathrm{Shi}^{4}$, Xiaoyan $\mathrm{Wu}^{3}$, Chang $\mathrm{Liu}^{2}$, Zhe Peng ${ }^{4}$, \\ Hong $\mathrm{Han}^{5}$, Shaojian $\mathrm{Xu}^{4}$, Ying Yang${ }^{2}$, Yao Tian ${ }^{2}$, JiaXin $\mathrm{Li}^{2}$, Hong-bin $\mathrm{He}^{6}$, and Jun $\mathrm{Li}^{4}$ \\ ${ }^{1}$ Affiliation not available \\ ${ }^{2}$ Division of Swine Diseases, Shandong Provincial Key Laboratory of Animal Disease \\ Control \& Breeding, Institute of Animal Science and Veterinary Medicine Shandong \\ Academy of Agricultural \\ ${ }^{3} 1$ Shandong Provincial Key Laboratory of Animal Disease Control \& Breeding, Institute \\ of Animal Science and Veterinary Medicine, Shandong Academy of Agricultural Sciences, \\ Jinan, China \\ ${ }^{4}$ Animal Science and Veterinary Medicine \\ ${ }^{5}$ Shandong Academy of Agricultural Sciences \\ ${ }^{6}$ Shandong Academy of Agricultural science
}

November 20, 2020

\begin{abstract}
Phospholipase C (PLC) is a key enzyme in the cell membrane. PLC hydrolyses phosphatidylinositol 4, 5-bisphosphate (PIP2) to generateinositol 1,4, 5-triphosphate (IP3) and diacylglycerol (DAG) that regulates a variety of cellular processes. Evidence indicates the pivotal role of PLC and inositol 1,4,5-trisphosphate receptor(IP3R) in influencing Ca2+ release from the endoplasmic reticulum(ER).At the same time, the imbalance of $\mathrm{Ca} 2+$ will stimulate endoplasmic reticulum stress(ERS), leading to cell apoptosis. Viral infection could triggers host defense through apoptosis of the infected cells. However, it is not clear how porcine circovirus type 2 (PCV2) induces apoptosis by affecting Ca2+ homeostasis. We show here that PCV2 infection induces the increased cytoplasmic Ca2+ level and apoptosis.We also found that the ER swelling of PK-15 cells after viral infection by transmission electron microscopy. Furthemore, the activation of PLC-IP3R-Ca2+ signaling enhanced apoptosis in infected PK-15 cells. Taken together,our findings suggest that PCV2 infection trigger ERS of PK-15 cells via the PLC-IP3R-Ca2+ signaling pathway to promoted the release of intracellular $\mathrm{Ca} 2+$, and led to cell apoptosis.
\end{abstract}

\section{Hosted file}

manuscripts_2020.11.19.pdf available at https://authorea.com/users/374885/articles/494096pcv2-trigger-apoptosis-of-pk-15-cells-through-the-plc-ip3r-ca2-signaling-pathway 

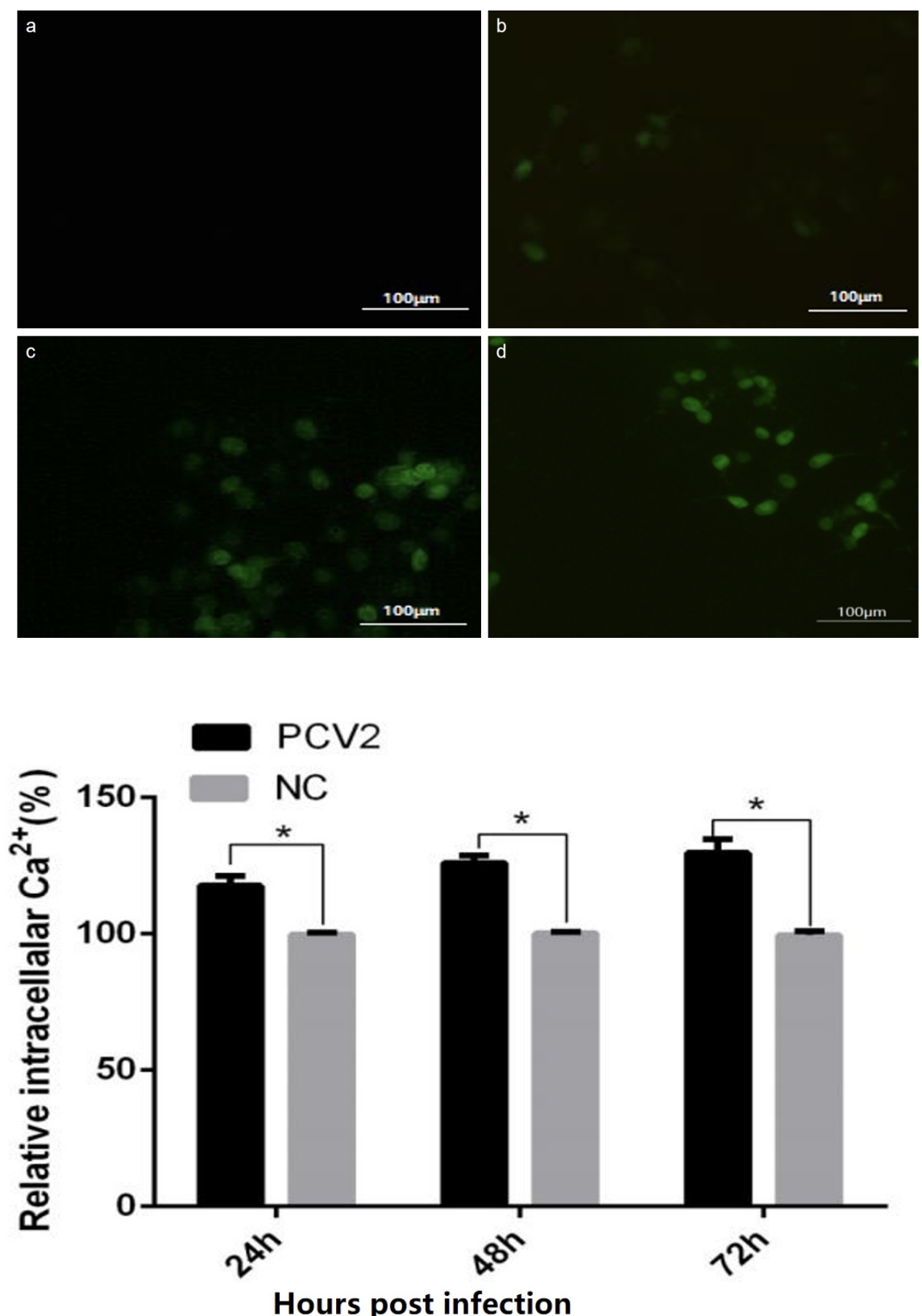

Hours post infection 

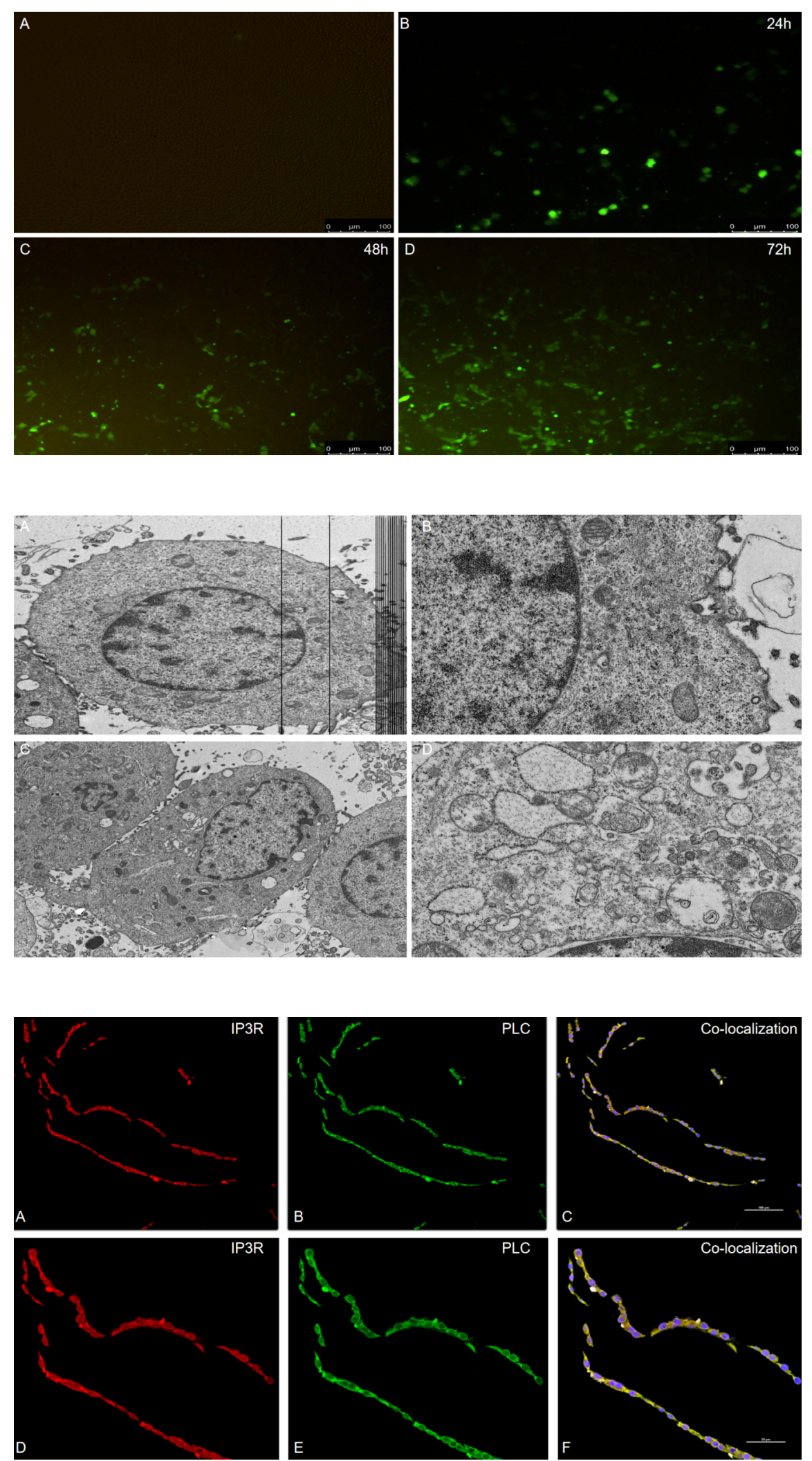

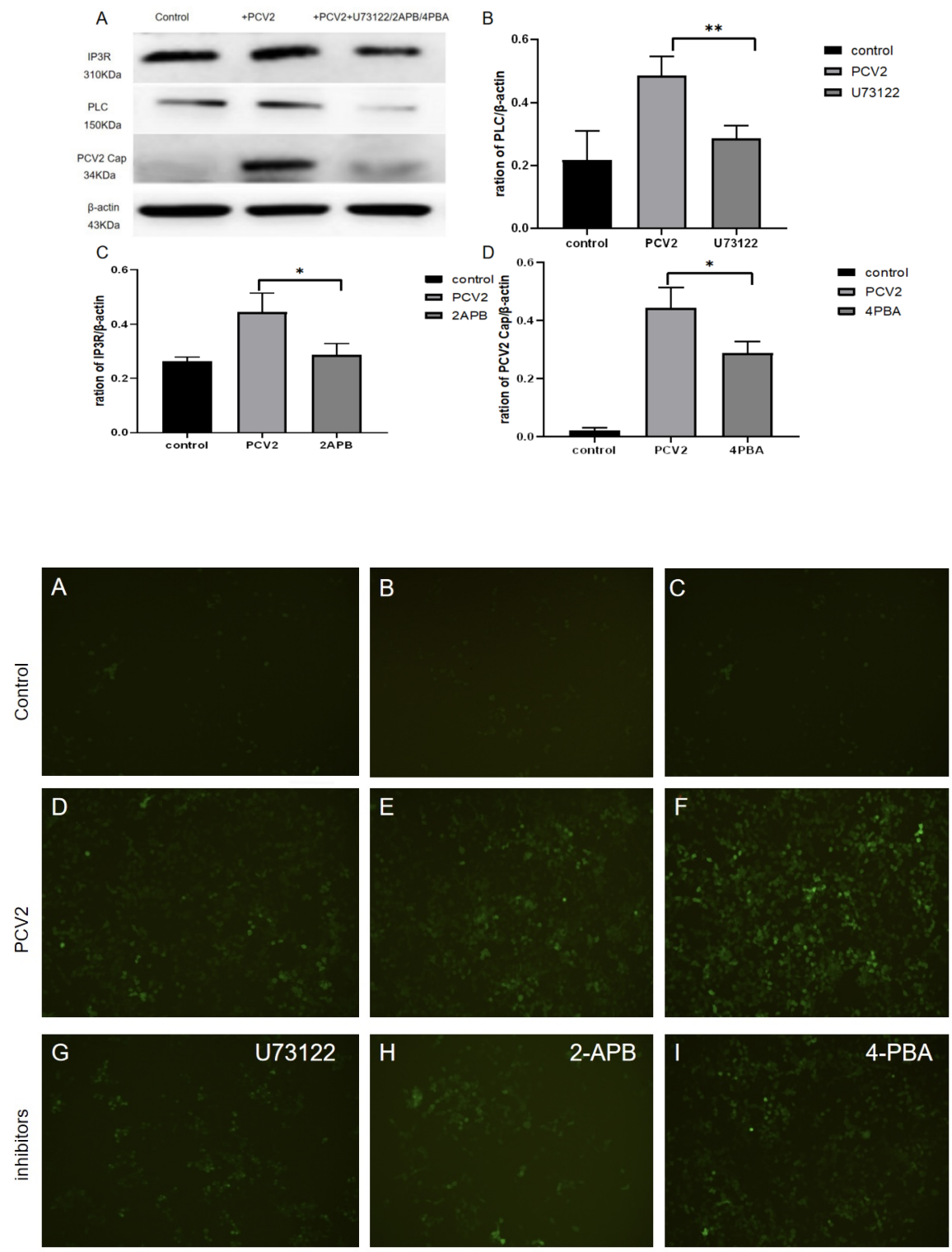

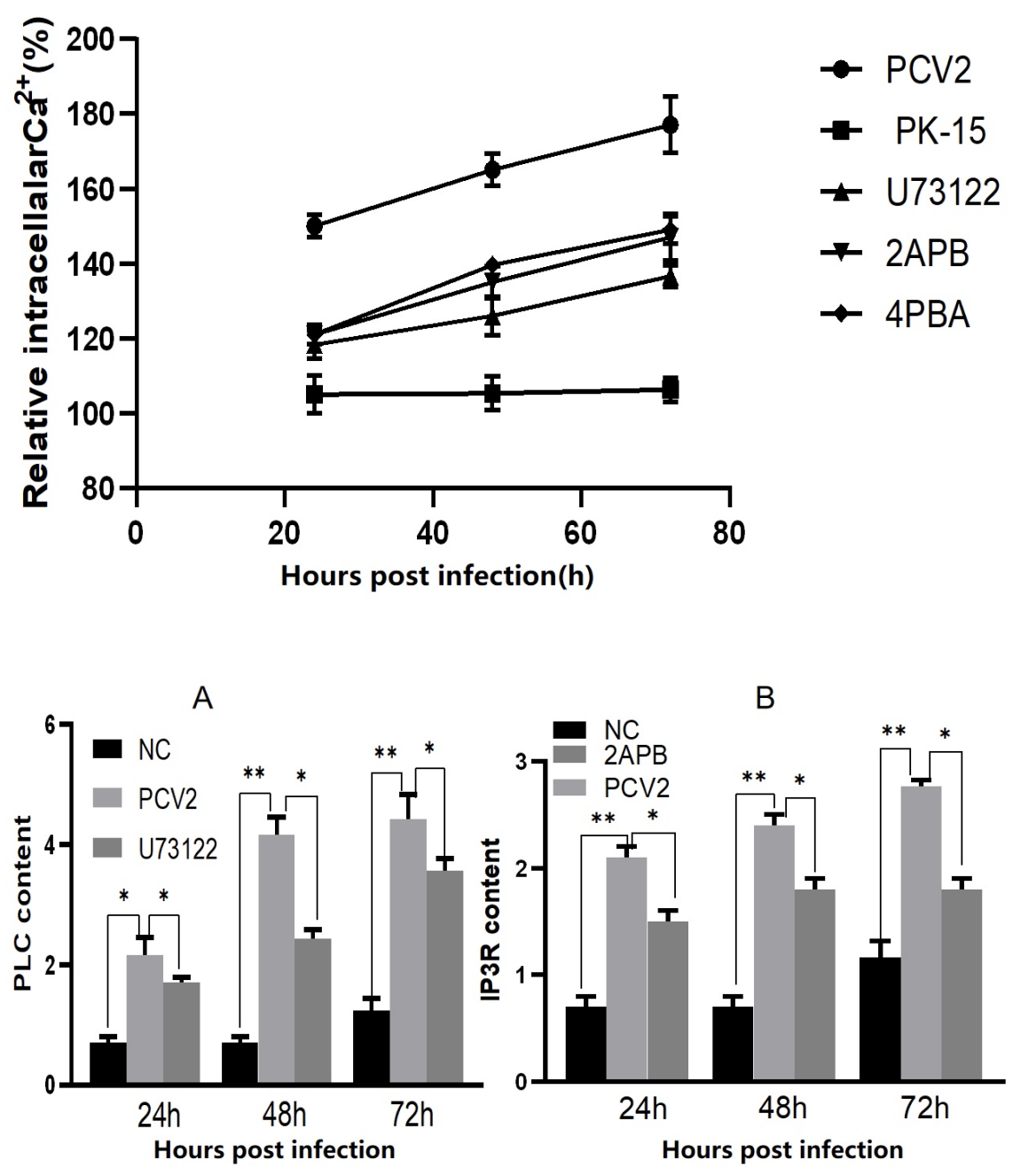